\title{
Assessing Pruritus in Hidradenitis Suppurativa: A Cross-Sectional Study
}

\author{
Allard R. J. V. Vossen $^{1}$ (D) Annelien Schoenmakers ${ }^{1} \cdot$ Kelsey R. van Straalen $^{1} \cdot$ \\ Errol P. Prens ${ }^{1} \cdot$ Hessel H. van der Zee ${ }^{1}$
}

Published online: 20 April 2017

(C) The Author(s) 2017. This article is an open access publication

\begin{abstract}
Background Pruritus is still a forgotten aspect of hidradenitis suppurativa (HS) and, to date, has never been adequately studied.

Objective The aim of this study was to determine the prevalence, and explore the characteristics, of pruritus in a well-defined cohort of HS patients.

Setting An academic hospital-based cross-sectional study in The Netherlands.

Methods A numerical rating scale (NRS, 0-10) was used to determine the prevalence of HS-related itch (NRS score $\geq 3$ ). Candidate predictors for pruritus were subsequently determined using logistic regression models, and the impact of pruritus was assessed using a modified five-dimensional (5-D) itch scale. Associated serological and histological markers of pruritus were (semi-)quantitatively investigated in a subpopulation.

Results The prevalence rate of pruritus in $211 \mathrm{HS}$ patients was $57.3 \%$, with a mean NRS score of $6.1 \pm 2.0$. Patients with a pruritus NRS score $\geq 3$ had more HS-affected body sites than patients with a score $<3 \quad(p<0.001)$. The occurrence of a pruritus NRS score $\geq 3$ was associated with
\end{abstract}

This work was presented at the 6th Conference of the European Hidradenitis Suppurativa Foundation, Copenhagen, Denmark, 8-10 February 2017.

Electronic supplementary material The online version of this article (doi:10.1007/s40257-017-0280-2) contains supplementary material, which is available to authorized users.

Allard R. J. V. Vossen

a.vossen@erasmusmc.nl

1 Department of Dermatology, Erasmus University Medical Center, Burgemeester s' Jacobplein 51, 3015 CA Rotterdam, The Netherlands
Hurley III disease (odds ratio [OR] 7.73; $p=0.003$ ) and pain (OR 1.34; $p<0.001$ ). Pruritus affected sleep and activities of daily living (ADL) in the majority of cases, with an associated modified 5-D itch score of $13.7 \pm 3.6$ (on a scale from 5 to 25) in 52 HS patients. Histological examination showed eosinophilic granulocytes were present in 25\% (2/8) of the perilesional skin and 63\% (10/16) of the lesional skin, while a perineural infiltrate was found in $25 \%(2 / 8)$ and $69 \%(11 / 16)$ of the perilesional and lesional skin, respectively.

Conclusion Pruritus is a frequent but underreported symptom in patients with HS. Its moderate to severe intensity and significant impact on daily activities have great potential to impair patients' quality of life.

\section{Key Points}

Pruritus or itch is a frequent and bothersome symptom in hidradenitis suppurativa (HS).

The presence of eosinophilic granulocytes and a perineural infiltrate in lesional skin might explain the reason for pruritus in HS.

The assessment of itch in both daily practice and clinical research settings may form a helpful additional patient-reported outcome measure to evaluate the disease activity/severity of HS.

\section{Introduction}

Hidradenitis suppurativa (HS), also known as acne inversa, is a chronic, recurrent, inflammatory skin disease that mostly develops after puberty and predominantly occurs in 
women, with a female-to-male ratio of $3: 1[1,2]$. The estimated prevalence rate in Europe is approximately $1 \%$ [1]. Risk factors for developing HS are smoking and overweight or obesity [3]. The disease is characterized by deep-seated, inflamed nodules and abscesses, often followed by sinus tract formation, and is most commonly located in the flexural body sites carrying terminal hairs [4].

Key symptoms of HS include acute and chronic pain, discomfort, and a purulent, foul-smelling discharge, which, overall, contribute to a poor quality of life $[5,6]$. Previous clinical studies have mainly focused on these well-known symptoms $[1,4,5]$, while less is known about the itch sensation, also known as pruritus. In 2011, a small qualitative study in which $12 \mathrm{HS}$ patients were interviewed for the first time revealed that not only drainage, pain, and scarring but also itching have a significant psychosocial impact in HS patients [7].

To date, one cross-sectional study demonstrated a positive association between disutility and itch in a cohort of 294 HS patients, using the EuroQoL-5D (EQ-5D) and a visual analog scale (VAS), respectively [8]. However, the prevalence and accompanying (psychosocial) factors of pruritus in HS have not been adequately investigated to date. The aim of this study was to determine the prevalence, and explore the characteristics, of pruritus in a cohort of HS patients. In addition, serological and histological markers of pruritus were evaluated in a subpopulation.

\section{Materials and Methods}

\subsection{Study Design and Population}

This cross-sectional study consisted of consecutive male and female patients with a physician-verified diagnosis of HS who visited the Department of Dermatology, Erasmus University Medical Center, Rotterdam, The Netherlands. Each patient filled out a questionnaire relating to the intensity of pruritus and the pain caused by their HS. Patients with a limited understanding of the Dutch language, as well as patients with a concomitant skin disease that might cause pruritus (e.g. psoriasis, atopic dermatitis, chronic urticaria) were excluded.

\subsection{Epidemiological and Clinical Parameters}

Patients separately assessed the highest intensity of their pruritus and pain over the past 7 days on an 11-point numerical rating scale (NRS) ranging from 0 (no itch/pain) to 10 (unbearable/extreme itch/pain) [9]. All patients with an NRS score $\geq 3$ were included in the prevalence analysis.
It has previously been demonstrated that the minimal clinically important difference (MCID) for clinical improvement in itch ranks as a decrease of 2.7 points rated on the NRS in the last 3 days [10].

Clinical parameters were collected during routine care and were derived from medical charts. HS severity was assessed using the Hurley classification of the worst affected body area [11], and the extent of disease activity was evaluated by the number of anatomical skin regions with inflammation. The presence of three or more papules/pustules, one or more inflammatory nodules, one or more draining sinuses, or one or more abscesses per region (left and right separate) was considered as inflammatory active HS disease. Additionally, the NRS relating to pain was used as the patient-reported outcome measure (PROM) of disease activity.

\subsection{The Impact of Pruritus}

Randomly selected patients with a pruritus NRS score $\geq 3$ were asked to fill in a five-dimensional (5-D) itch scale, which is used to evaluate the impact of pruritus on daily activities and includes five domains-duration, degree, direction, disability, and distribution [12].The questionnaire was adapted through (1) translation into Dutch, and (2) explicitly mentioning the axillary and genital regions, with removal (i.e. points of contact with clothing) and merging (i.e. tops of feet and soles; palms and tops of hands; thighs and lower legs; forearms and upper arms) of non-relevant sections in the distribution domain. The latter was carried out to obtain better insight into the HS predilection areas, and resulted in 12, instead of 16, answer options (electronic supplementary material 1). The outcomes of domains $1-4$ are measured on a 5-point Likert scale [12]. The scoring system for the distribution domain was not adapted as it was not expected that HS patients without concomitant skin disease that might cause pruritus would have more than 12 skin regions with symptoms of itch. The overall 5-D score was calculated by adding up the individual scores of the five domains, resulting in scores ranging from 5 (no impact) to 25 (most severe impact on daily life) [12].

\subsection{Serological Analysis}

A random subset of HS patients aged $\geq 18$ years with a pruritus NRS score $\geq 3$ was asked for a one-time collection of a blood sample in order to screen for other possible causes of pruritus [13, 14].Tryptase, hemoglobin, bilirubin, creatinine, urea, thyroid-stimulating hormone (TSH), and glycated hemoglobin (HbAlc) were assessed in serum. 


\subsection{Histological Analysis}

Histopathological analysis was performed on 24 random HS skin samples (including eight perilesional skin samples, and six early and ten chronic lesions) in order to evaluate potential skin-related mediators of pruritus. Large specimens of chronic inflamed skin were obtained from the excised skin of patients who had radical excision of their HS under general anesthesia as this was considered waste material. In our clinic, HS lesions are excised with a healthy-appearing skin margin of $2 \mathrm{~cm}$; this normal-appearing skin is denoted as perilesional skin. All early (i.e. newly emerging) HS lesions, as judged by both the HS patient and the dermatologist, were biopsied within 4 days after onset.

Three independent observers (ARJVV, KRvS, and EPP), blinded to the disease stage, assessed all skin samples, stained by hematoxylin and eosin (H\&E), on the density of the infiltrate, followed by evaluation of eosinophilic granulocytes and perineural involvement within the infiltrate. The outcomes were scored in a semiquantitative manner using a global assessment on an ordinal scale from 0 to 3 $(0=$ none, $1=$ mild, $2=$ moderate, $3=$ severe $)$ [15]. Disagreements between the three observers were resolved through discussion until consensus was reached. The average overall score per category was calculated from the consensus score of the observers.

\subsection{Statistical Analysis}

Statistical analyses were conducted using SPSS Statistics 21.0 (IBM Corporation, Armonk, NY, USA). Patient characteristics were analyzed using descriptive statistics, with continuous data presented as the mean \pm standard deviation (SD) or mean and interquartile range (IQR), and categorical data presented as number (\%). A ShapiroWilk test was performed to test whether continuous data were normally distributed. For the primary objective, i.e. the evaluation of patient characteristics between patients with and without pruritus, the parametric $t$ test for normally distributed independent samples (two-sided), the nonparametric Mann-Whitney U test for non-normally distributed samples, and the Chi-square test or Fisher's exact test for categorical data were applied. Candidate predictors for pruritus, based on both clinical experience and the literature, were subsequently determined using both univariable and multivariable logistic regression models, with pruritus (NRS score $\geq 3$ ) as the dependent variable. In order to prevent overfitting of the regression analysis, we were restricted to using 9 degrees of freedom in the multivariate model, as the limiting sample size of patients without pruritus was 90 (Table 1) [15]. Data were presented as odds ratio (OR) with $95 \%$ confidence intervals (CIs). In all comparisons, a two-sided $p$ value of 0.05 was considered significant.

\subsection{Ethical Statement}

The Medical Ethical Committee of the Erasmus University Medical Center, Rotterdam, The Netherlands, reviewed and approved the study protocol (reference MEC-2016092). Written informed consent for the serological and histological analysis was obtained from all subjects in accordance with the Declaration of Helsinki principles.

\section{Results}

\subsection{Epidemiology of Pruritus}

A total of 231 HS patients were screened, of whom 20 were excluded (13 had a concomitant dermatological comorbidity causing itch, 5 had a limited understanding of the Dutch language, and 2 patients declined to take part in the study). The 211 patients included in the study (64\% female) had a median age of 38 years (range 15-71) (Table 1). The prevalence rate (NRS score $\geq 3$ ) of pruritus was $57.3 \%$ (121/211), with a mean NRS score of $6.1 \pm 2.0$. For comparison, applying a cut-off value of an NRS score $\geq 1$ resulted in a rate of $67.3 \%$ (mean NRS score of $5.4 \pm 2.5$ ). The mean intensity of itch in all 211 patients was rated at an NRS score of $3.7 \pm 3.3$. The occurrence of pain (NRS score $\geq 3$ ) was more frequently reported, resulting in a prevalence rate of $83.4 \%$ and associated mean NRS score of $7.4 \pm 1.8$.

\subsection{Patient Characteristics}

Comparison of the patient characteristics between patients with pruritus (NRS score $\geq 3$ ) and patients reporting no or negligible pruritus (NRS score $<3$ ) revealed no significant differences, with the exception of HS disease activity/severity (Table 1). Patients with a pruritus NRS score $\geq 3$ reported a higher level of pain $(p<0.001)$, had more affected body areas $(p<0.001)$, and had more severe disease according to the Hurley classification $(p<0.001)$ compared with patients reporting a pruritus NRS score $<3$ (Table 1). Candidate predictors for patients reporting a pruritus NRS score $\geq 3$ were Hurley stage III (OR 7.73; $p=0.003)$ and pain, with an OR of 1.34 for each additional point on the NRS $(p<0.001)$ (Table 2). A higher number of currently inflamed areas was only associated with pruritus in the univariable analysis (OR 1.70; $p<0.001)$. In addition, patients who smoked tended to complain more about itch than patients who have never smoked (univariable analysis; OR 2.27; $p=0.03$ ). 
Table 1 Patient characteristics

\begin{tabular}{|c|c|c|c|c|}
\hline & Total $[n=211]$ & Pruritus NRS score $\geq 3[n=121]$ & Pruritus NRS score $<3[n=90]$ & $p$ value \\
\hline Age, years & $38.0(29-49)$ & $38.0(29-48)$ & $39.0(29-52)$ & 0.39 \\
\hline Female sex & $135(64)$ & $78(65)$ & $57(63)$ & 0.87 \\
\hline BMI, $\mathrm{kg} / \mathrm{m}^{2}$ & $28.5 \pm 5.9$ & $28.7 \pm 5.8^{\mathrm{a}}$ & $28.3 \pm 6.0^{\mathrm{b}}$ & 0.67 \\
\hline Diabetes mellitus & $18(9)$ & $10(8)$ & $8(9)$ & 0.87 \\
\hline Smoking status ${ }^{\mathrm{c}}$ & & & & 0.05 \\
\hline Never smoked & $37(20)$ & $16(13)$ & $21(23)$ & \\
\hline Present smoker & $42(62)$ & $83(70)$ & $48(54)$ & \\
\hline Past smoker & $131(18)$ & $21(18)$ & $21(23)$ & \\
\hline Pack-years $^{\mathrm{d}}$ & $12.0(7-23)$ & $12.9(6-22)$ & $12.0(7-26)$ & 0.65 \\
\hline Positive family history ${ }^{\mathrm{e}}$ & $80(38)$ & $49(41)$ & $31(34)$ & 0.37 \\
\hline Use of systemic medication & $64(32)$ & $36(30)$ & $28(31)$ & 0.92 \\
\hline Skin type $(\text { Fitzpatrick })^{\mathrm{f}}$ & & & & 0.49 \\
\hline I & $18(9)$ & $14(12)$ & $4(5)$ & \\
\hline II & $126(60)$ & $69(57)$ & $57(63)$ & \\
\hline III & $17(8)$ & $11(9)$ & $6(7)$ & \\
\hline IV & $30(14)$ & $17(14)$ & $13(14)$ & \\
\hline $\mathrm{V}$ & $11(5)$ & $5(4)$ & $6(7)$ & \\
\hline VI & $9(4)$ & $5(4)$ & $4(4)$ & \\
\hline Duration of HS, years & $14.0(7-25)$ & $15.0(8-27)$ & $13.0(5-23)$ & 0.22 \\
\hline HS severity ${ }^{\mathrm{g}}$ & & & & $<0.001 * *$ \\
\hline Hurley I & $32(15)$ & $13(11)$ & $19(21)$ & \\
\hline Hurley II & $140(66)$ & $75(62)$ & $65(72)$ & \\
\hline Hurley III & 39 (19) & $33(27)$ & $6(7)$ & \\
\hline Currently inflamed areas ${ }^{\mathrm{h}}$ & $1.5 \pm 1.3$ & $1.7 \pm 1.4$ & $0.9 \pm 1.1$ & $<0.001 * *$ \\
\hline Pain on NRS & $6.3 \pm 3.0$ & $6.9 \pm 2.5$ & $5.0 \pm 3.5$ & $<0.001 * *$ \\
\hline
\end{tabular}

Data are expressed as mean $\pm \mathrm{SD}$, median (IQR), or $n(\%)$

$B M I$ body mass index, $H S$ hidradenitis suppurativa, $I Q R$ interquartile range, $N R S$ numerical rating scale, $S D$ standard deviation

* Indicates significant at $p=0.05, * *$ indicates significant at $p=0.001$

${ }^{\text {a }}$ Missing $n=6$

b Missing $n=9$

c Missing $n=1$

${ }^{\mathrm{d}}$ Pack-year $=($ number of cigarettes smoked per day $\times$ number of years smoked $) / 20$

e A positive family history for HS symptoms in first- and second-degree relatives

${ }^{\mathrm{f}}$ Fisher's exact test

g Chi-square test

${ }^{\mathrm{h}}$ Inflamed area $=$ three or more papules/pustules, one or more inflammatory nodules, one or more draining sinuses, or one or more abscesses

\subsection{The Impact of Pruritus}

Fifty-two patients in the pruritus NRS score $\geq 3$ group $(n=121)$ completed the 5-D itch scale; however, one incorrectly filled out questionnaire was excluded from the analysis. First, the most commonly reported pruritus characterization was moderate $(54 \%)$ to severe $(27 \%)$ itching sensations for less than $6 \mathrm{~h}$ per day $(56 \%)$, which had not changed in the previous 2 weeks $(48 \%)$. Second, pruritus, at least occasionally, affected sleep and activities of daily living (ADL), i.e. leisure, social contact, housework, errands, and work/school, in 70 and $53 \%$ of patients, respectively (Fig. 1a). The mean number of body areas affected by pruritus was $3.1 \pm 2.5$, with the inguinal/genital $(67 \%)$ and axillary (52\%) regions most frequently involved (Fig. 1b). The overall modified 5-D itch score was $13.7 \pm 3.6$, i.e. HS-related pruritus had a moderate impact on daily activities. 
Table 2 Associations between patient characteristics and the occurrence of itch in $211 \mathrm{HS}$ patients

\begin{tabular}{|c|c|c|c|c|}
\hline \multirow[t]{2}{*}{ Coding } & \multicolumn{2}{|l|}{ Univariable $^{\mathrm{a}}$} & \multicolumn{2}{|l|}{ Multivariable $^{a}$} \\
\hline & OR $(95 \% \mathrm{CI})$ & $p$ value & OR $(95 \% \mathrm{CI})$ & $p$ value \\
\hline \multicolumn{5}{|l|}{ Age } \\
\hline Continuous & $0.99(0.97-1.01)$ & 0.33 & $0.97(0.94-1.01)$ & 0.10 \\
\hline \multicolumn{5}{|l|}{ Sex } \\
\hline Male & $1.05(0.60-1.86)$ & 0.87 & $0.75(0.37-1.53)$ & 0.75 \\
\hline \multicolumn{5}{|l|}{ Smoking status } \\
\hline Never smoked & Reference variable & & Reference variable & \\
\hline Present smoker & $2.27(1.08-1.76)$ & $0.03 *$ & $2.02(0.82-4.95)$ & 0.13 \\
\hline Past smoker & $1.31(0.54-3.19)$ & 0.55 & $1.26(0.43-3.68)$ & 0.67 \\
\hline \multicolumn{5}{|c|}{ Duration of HS (years) } \\
\hline Continuous & $1.02(0.99-1.04)$ & 0.20 & $1.04(1.00-1.07)$ & 0.38 \\
\hline \multicolumn{5}{|l|}{ HS severity } \\
\hline Hurley I & Reference variable & & Reference variable & \\
\hline Hurley II & $1.69(0.77-3.68)$ & 0.19 & $1.78(0.72-4.45)$ & 0.22 \\
\hline Hurley III & $8.04(2.62-25)$ & $<0.001 * *$ & $7.73(2.01-27)$ & $0.003 *$ \\
\hline \multicolumn{5}{|c|}{ Currently inflamed areas } \\
\hline Continuous & $1.70(1.32-2.19)$ & $<0.001 * *$ & $1.21(0.92-1.58)$ & 0.18 \\
\hline \multicolumn{5}{|l|}{ Pain on NRS } \\
\hline Continuous & $1.35(1.12-1.50)$ & $<0.001 * *$ & $1.34(1.18-1.52)$ & $<0.001 * *$ \\
\hline $\begin{array}{l}H S \text { hidradenitis } \mathrm{s} \\
* \text { Indicates signif }\end{array}$ & $\begin{array}{l}J R S \text { numerical rating } \\
0.05, * * \text { indicates si }\end{array}$ & $\begin{array}{l}\text { le, } O R \text { odd } \\
\text { icant at } p=\end{array}$ & $\begin{array}{l}\text { io, } C I \text { confidence int } \\
01\end{array}$ & \\
\hline
\end{tabular}

\subsection{Serological Analysis}

Serum pruritus markers were evaluated in 24 patients in the pruritus NRS score $\geq 3$ group $(n=121)$. The mean common serum values were within the normal range, with the exception of a lower hemoglobin level found in male patients $(n=5)$ (Table 3$)$. Three outliers were detected: tryptase $(19.3 \mu \mathrm{gr} / \mathrm{L})$ in one patient with a pruritus NRS score of 7, and HbAlc (91.0 and $57.0 \mathrm{mmol} / \mathrm{mol}$ ) in two patients with pruritus NRS scores of 3 and 5, respectively.

\subsection{Histological Analysis}

The average semiquantitative scores for the three types of HS skin specimens are presented in Fig. 2. Histological evaluation showed that inflammatory cell infiltration was present (i.e. score of 1-3) in 75\% (6/8) of the perilesional samples, and $100 \%(16 / 16)$ of both early and chronic lesional samples. Eosinophilic granulocytes were present (i.e. score of 1-3) in $25 \%$ (2/8) of perilesional skin and $63 \%(10 / 16)$ of lesional skin (Fig. 3a). A perineural infiltrate, mainly consisting of lymphocytes and a few neutrophils, was found in 25\% (2/8) and 69\% (11/16) of perilesional and lesional skin, respectively (Fig. 3b).

\section{Discussion}

For the first time, this explorative study evaluated the prevalence and clinical characteristics of pruritus in a welldefined cohort of HS patients. We demonstrated a high prevalence rate of $57 \%$ (121/211 with an NRS score $\geq 3$ ), with a mean NRS score of $6.1 \pm 2.0$. This prevalence rate is substantially higher than the prevalence of itch in a healthy French population (29\%) and a Norwegian national sample with several ethnicities (7\%) $[16,17]$. A recently conducted study involving a general dermatological population of a German clinical practice reported a $36 \%$ prevalence rate of itch [18]. Compared with other inflammatory skin conditions, the $57 \%$ prevalence rate of pruritus in HS is similar to the rate in patients with psoriasis (49-90\%) [18, 19], and lower than patients with burn injuries $(67-93 \%)[20,21]$ or chronic idiopathic urticaria (79\%) [22]. 
Fig. 1 The Modified

5-Dimensional Itch Scale of 52 hidradenitis suppurativa patients with a pruritus numerical rating scale score of $\geq 3$. (a) Disability: the impact of itch on sleep and ADL in the past 2 weeks.

(b) Distribution of itch per body area in the past 2 weeks. $A D L$ activities of daily living, based on the highest outcome of the three subcategories in the questionnaire

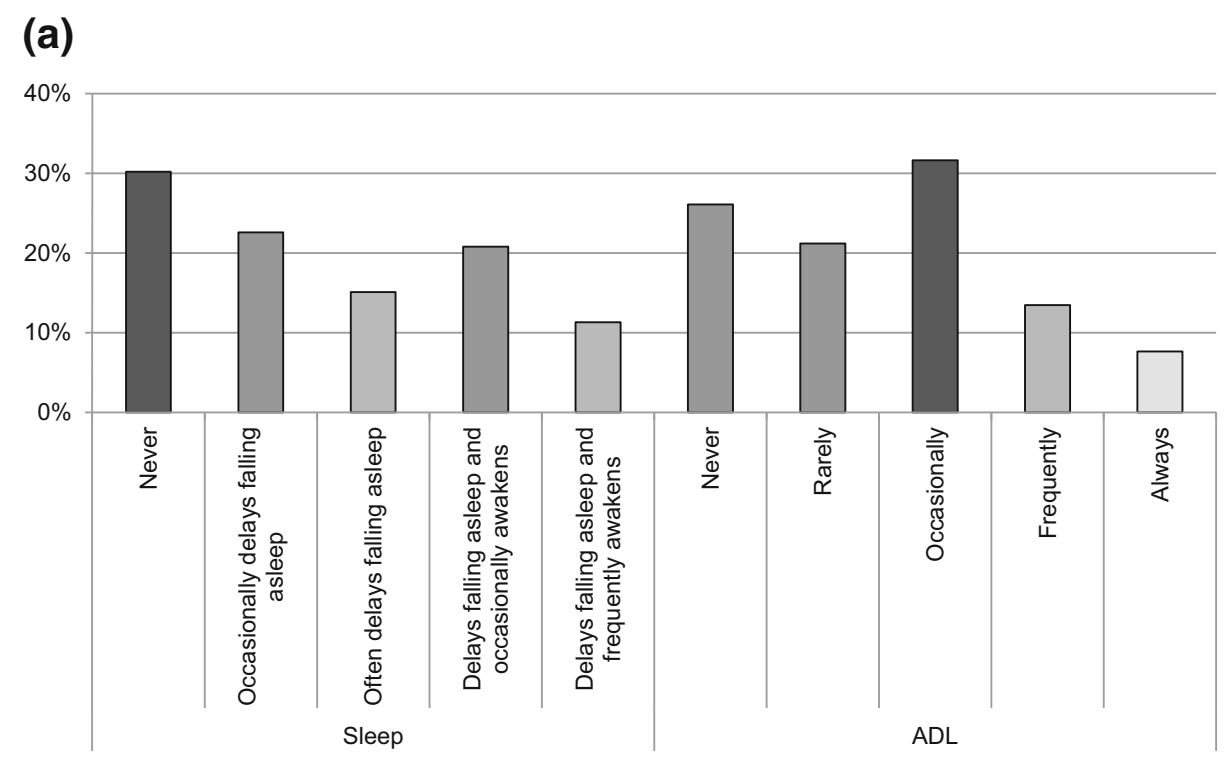

(b)

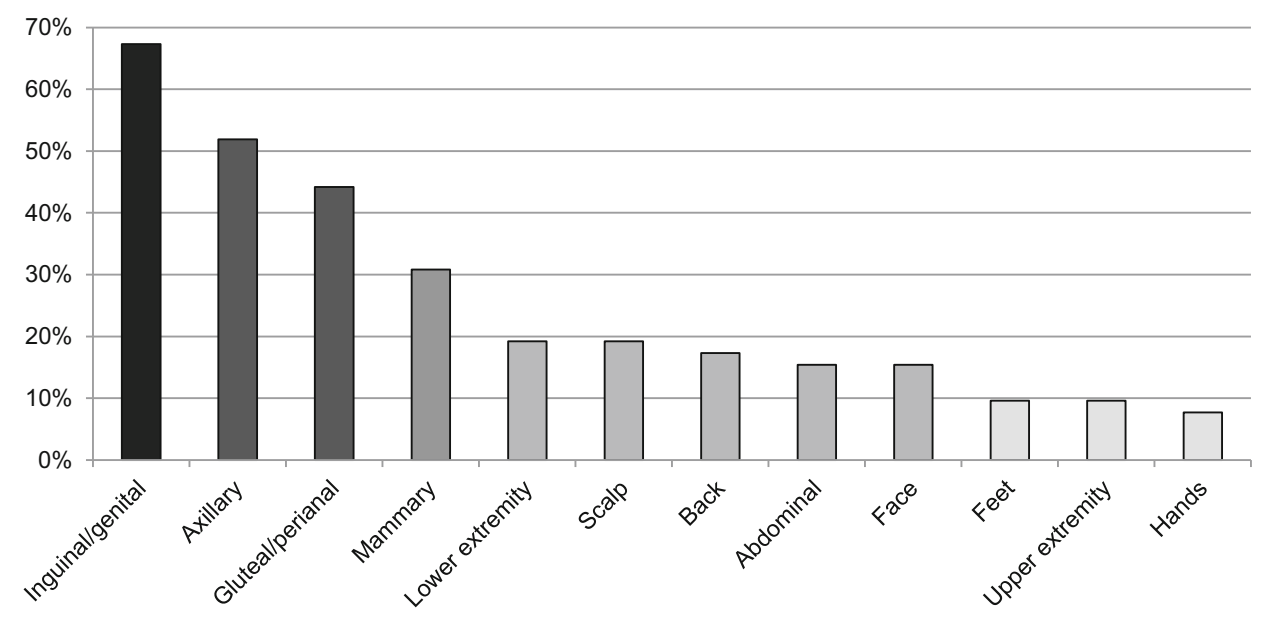

The overall mean intensity of itch in this study (NRS score $3.7 \pm 3.3$ ) was similar to the pruritus VAS score of $3.7 \pm 3.2$ in a cohort of 294 HS patients [8]. In addition, the mean modified 5-D itch score of $13.7 \pm 3.6$ in $52 \mathrm{HS}$ patients is comparable with the 5-D score in 51 patients with an inflammatory skin condition such as burn wounds $(13.5 \pm 3.2)$ [12]. Systemic diseases such as HIV/AIDS $(n=28 ; 16.8 \pm 5.3)$, chronic liver disease $(n=63$; $16.9 \pm 4.7)$, and chronic kidney disease $(n=36$; $18.2 \pm 4.1)$ have shown higher 5-D itch scores [12]; however, the majority $(81 \%)$ of HS patients ranked the severity of pruritus as moderate to severe on a 5-point Likert scale. Moreover, sleep and ADL were negatively impacted by pruritus in more than half of the patients.
Pruritus is a multidimensional phenomenon and is thought to signal danger from various environmental factors or physiological abnormalities. Therefore, it frequently accompanies various inflammatory skin conditions, including atopic dermatitis, psoriasis, chronic urticaria, and burn wound healing [23].To date, the etiology of pruritus in skin diseases is only partially understood. A possible explanation for itching in HS patients is the presence of tryptase-positive mast cells, which were found to be increased in all stages of the disease, including perilesional skin [24]. Increased serum levels of immunoglobulin (Ig)E have recently been reported in patients with HS. The latter, in combination with a dense infiltration of mast cells in HS, could trigger degranulation of these cells, releasing 
Table 3 Serum pruritus markers of 24 hidradenitis suppurativa patients with itch

\begin{tabular}{llcl}
\hline Serum pruritus marker & Units & Mean value & Reference \\
\hline Bilirubin & $\mu \mathrm{mol} / \mathrm{L}$ & $6.0 \pm 2.6$ & $<17$ \\
Creatinine & $\mu \mathrm{mol} / \mathrm{L}$ & $69.4 \pm 16.8$ & $55-115^{\mathrm{a}}$ \\
HbA1c & $\mathrm{mmol} / \mathrm{mol}$ & $38.9 \pm 12.7$ & $26-42$ \\
Hemoglobin & & & \\
$\quad$ Men $(n=5)$ & $\mathrm{mmol} / \mathrm{L}$ & $8.4 \pm 1.2$ & $8.6-10.5$ \\
Women $(n=19)$ & $\mathrm{mmol} / \mathrm{L}$ & $8.4 \pm 0.6$ & $7.5-9.5$ \\
Tryptase & $\mu \mathrm{gr} / \mathrm{L}$ & $5.4 \pm 3.5$ & $<11.4$ \\
TSH & $\mathrm{mU} / \mathrm{L}$ & $1.6 \pm 1.0$ & $0.4-4.3$ \\
Urea $^{\mathrm{b}}$ & $\mathrm{mmol} / \mathrm{L}$ & $4.0 \pm 1.5$ & $2.5-7.5$ \\
\hline
\end{tabular}

HbAlc glycated hemoglobin, TSH thyroid-stimulating hormone

${ }^{\text {a }}$ Upper limit dependent on ethnic origin, sex and age

b $n=1$ missing

histamine and other mediators, such as proteases, causing pruritus [25]. In addition, we found an influx of eosinophilic granulocytes and the presence of a perineural infiltrate (when nerve fibers were present) in the majority of prototypic HS lesions. An important phenomenon supporting the hypothesis of neurogenic inflammation is the finding of an abnormal innervation of the skin in psoriasis [26]. As demonstrated in cutaneous T-cell lymphoma, interleukin (IL)-31 may play a role by exerting indirect effects on sensory nerves through keratinocytes to transmit itch signals [27]. However, itch in HS patients could also result from a small fiber neuropathy due to scar formation in the course of HS. Recurrent and chronic inflammation will destroy dermal nerves and subsequently enhance nerve regeneration and neovascularization when inflammation has subsided. This type of neuropathic itch coincides with pain and may be caused by a disproportionate number of regenerating, unmyelinated $\mathrm{C}$ nerve fibers within $\mathrm{HS}$ lesions [28].

The major strength of this explorative cross-sectional study is the recruitment of a relatively large number of physician-verified HS patients. As the occurrence of itch at a specific point in time is susceptible to confounders such as mental distress, use of systemic medication, and comorbidities [29], a comparator group and quality-of-life measurements, e.g. Dermatology Quality of Life Index (DLQI) or SKINDEX-29, are indicated for future research. Another strength is screening for common systemic causes of chronic recurrent itch that could interfere with the occurrence of HS-related itch. In addition, histopathological evaluation of two important skin-related mediators of pruritus have been performed, although the evaluation lacks information relating to the associated presence of pruritus. For future analysis, it would be interesting to evaluate specific itch-related biomarkers in the serum or skin lesions, such as eosinophilic granulocytes, thymus and activation-regulated chemokine (TARC), IL-2, IL31 , and substance $P$.

A limitation of this study could be the use of a nonvalidated, HS-modified 5-D itch scale. From a clinical point of view, the alteration of the distribution section was not expected to influence scoring as HS patients rarely have notable itch in distal, unaffected body sites such as the hands and feet. Moreover, the body areas mentioned in the distribution domain of the adapted scale are more specific to HS-related itch (electronic supplementary material 1). The modified 5-D itch scale, which could be validated for this application in future research, could therefore be a specialized and very useful tool for analysing pruritus in HS patients.

\section{Conclusion}

Pruritus is a frequent but underreported aspect of HS. Its moderate to severe intensity and significant impact on sleep and ADL have great potential to impair patients' quality of life. Therefore, assessment of pruritus in both daily practice and clinical research settings, e.g. by using
Fig. 2 Semiquantitative scores for histological markers of pruritus in three types of hidradenitis suppurativa lesions. A total of 24 samples were assessed: perilesional, $n=8$; early lesional, $n=6$; chronic lesional, $n=10$. The $\mathrm{y}$-axis indicates the average semiquantitative scores on an ordinal scale from 0 to 3 $(0=$ none, $1=$ mild,

$2=$ moderate, $3=$ severe)

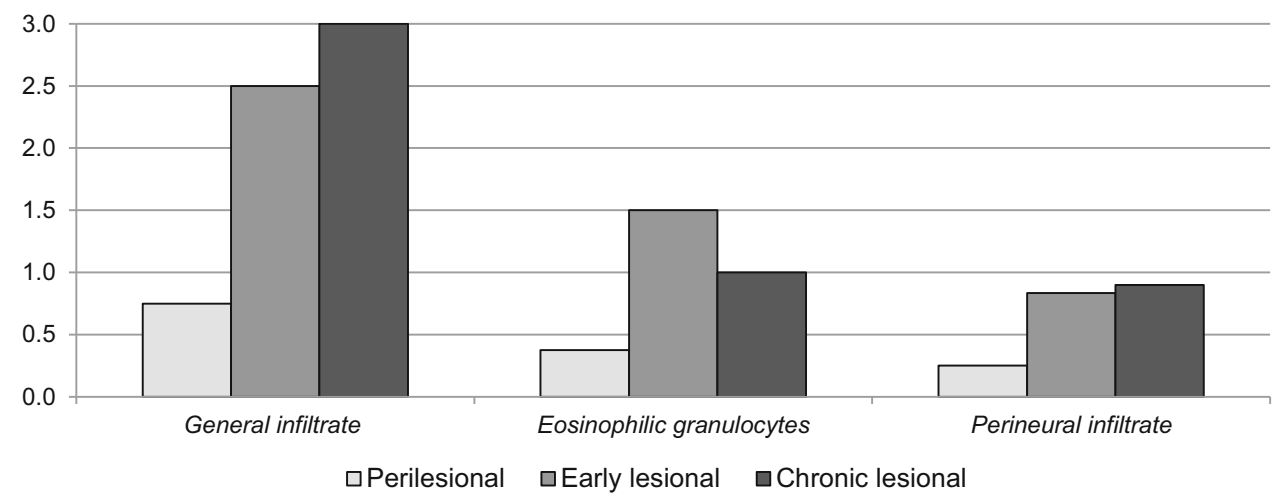



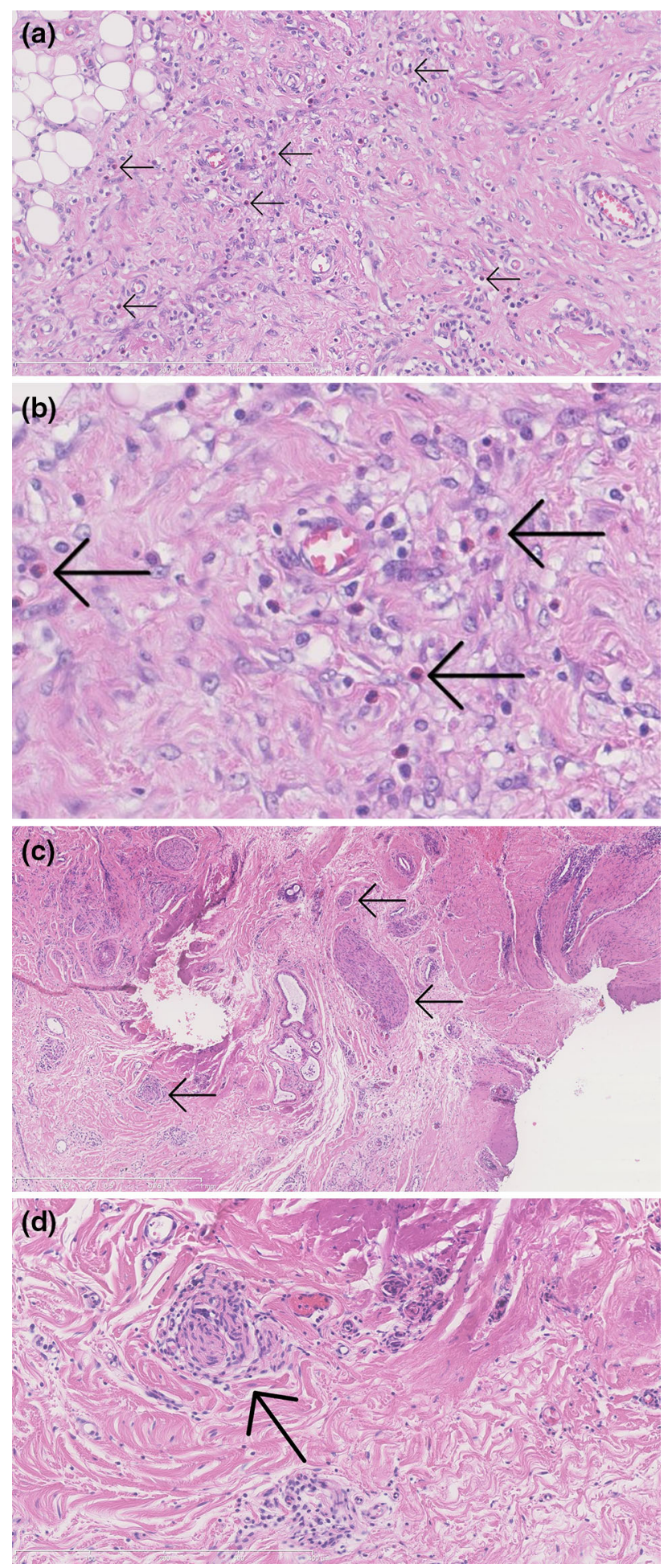

Fig. 3 Two histological pruritus markers in hidradenitis suppurativa lesional skin. (a) Inflammatory infiltrate with influx of eosinophilic granulocytes (arrows); H\&E, $\times 20$. (b) Closer view of above. (c) Perineural infiltration of the peripheral nerves (arrows) in overview; H\&E, $\times 5$. (d) Infiltration of a peripheral nerve (arrow) by lymphocytes and a few neutrophils in detail; $H \& E, \times 20 . H \& E$ hematoxylin and eosin an NRS or VAS, together with the DLQI and EQ-5D, may form a helpful additional PROM to evaluate disease severity/activity and treatment outcome.

Acknowledgments The authors thank Loes Hollestein, Epidemiologist, Department of Dermatology, Erasmus University Medical Center, for her assistance in the statistical analysis.

\section{Compliance with Ethical Standards}

Funding No sources of funding were used to assist in the preparation of this article.

Conflicts of interest Allard R.J.V. Vossen, Annelien Schoenmakers, Kelsey R. van Straalen, Errol P. Prens, Hessel H. van der Zee have no conflicts of interest to declare.

Ethical approval Ethical approval was provided by the Institutional Review Board of the Erasmus University Medical Center.

Statistics Statistical methods and tests were approved by Loes Hollestein (PhD), Epidemiologist, Department of Dermatology, Erasmus University Medical Center, Rotterdam, The Netherlands.

Open Access This article is distributed under the terms of the Creative Commons Attribution-NonCommercial 4.0 International License (http://creativecommons.org/licenses/by-nc/4.0/), which permits any noncommercial use, distribution, and reproduction in any medium, provided you give appropriate credit to the original author(s) and the source, provide a link to the Creative Commons license, and indicate if changes were made.

\section{References}

1. Zouboulis CC, Desai N, Emtestam L, et al. European S1 guideline for the treatment of hidradenitis suppurativa/acne inversa. J Eur Acad Dermatol Venereol. 2015;29:619-44.

2. Miller IM, McAndrew RJ, Hamzavi I. Prevalence, risk factors, and comorbidities of hidradenitis suppurativa. Dermatol Clin. 2016;34:7-16.

3. Sartorius K, Emtestam L, Jemec GB, et al. Objective scoring of hidradenitis suppurativa reflecting the role of tobacco smoking and obesity. Br J Dermatol. 2009;161:831-9.

4. Jemec GB. Clinical practice. Hidradenitis suppurativa. N Engl J Med. 2012;366:158-64.

5. Dufour DN, Emtestam L, Jemec GB. Hidradenitis suppurativa: a common and burdensome, yet under-recognised, inflammatory skin disease. Postgrad Med J. 2014;90:216-21 (quiz 20).

6. Matusiak L, Bieniek A, Szepietowski JC. Hidradenitis suppurativa markedly decreases quality of life and professional activity. J Am Acad Dermatol. 2010;62:706-8 (708.e1).

7. Esmann S, Jemec GB. Psychosocial impact of hidradenitis suppurativa: a qualitative study. Acta Derm Venereol. 2011;91:328-32.

8. Riis PT, Vinding GR, Ring HC, et al. Disutility in patients with hidradenitis suppurativa: a cross-sectional study using EuroQoL5D. Acta Derm Venereol. 2016;96:222-6.

9. Phan NQ, Blome C, Fritz F, et al. Assessment of pruritus intensity: prospective study on validity and reliability of the visual analogue scale, numerical rating scale and verbal rating scale in 
471 patients with chronic pruritus. Acta Derm Venereol. 2012;92:502-7.

10. Reich A, Riepe C, Anastasiadou Z, et al. Itch assessment with visual analogue scale and numerical rating scale: determination of minimal clinically important difference in chronic itch. Acta Derm Venereol. 2016;96:978-80.

11. Revuz J. Hidradenitis suppurativa. J Eur Acad Dermatol Venereol. 2009;23:985-98.

12. Elman S, Hynan LS, Gabriel V, et al. The 5-D itch scale: a new measure of pruritus. Br J Dermatol. 2010;162:587-93.

13. Pereira MP, Kremer AE, Mettang T, et al. Chronic pruritus in the absence of skin disease: pathophysiology, diagnosis and treatment. Am J Clin Dermatol. 2016;17(4):337-48.

14. Yosipovitch G, Greaves MW, Schmelz M. Itch. Lancet. 2003;361:690-4

15. Whitehead J. Sample size calculations for ordered categorical data. Stat Med. 1993;12:2257-71.

16. Dalgard F, Holm JO, Svensson A, et al. Self reported skin morbidity and ethnicity: a population-based study in a Western community. BMC Dermatol. 2007;7:4.

17. Misery L, Rahhali N, Duhamel A, et al. Epidemiology of pruritus in France. Acta Derm Venereol. 2012;92:541-2.

18. Kopyciok ME, Stander HF, Osada N, et al. Prevalence and characteristics of pruritus: a one-week cross-sectional study in a German dermatology practice. Acta Derm Venereol. 2016;96:50-5.

19. Remrod C, Sjostrom K, Svensson A. Pruritus in psoriasis: a study of personality traits, depression and anxiety. Acta Derm Venereol. 2015;95:439-43.

20. Van Loey NE, Bremer M, Faber AW, et al. Itching following burns: epidemiology and predictors. $\mathrm{Br} \mathrm{J}$ Dermatol. 2008;158:95-100.
21. Carrougher GJ, Martinez EM, McMullen KS, et al. Pruritus in adult burn survivors: postburn prevalence and risk factors associated with increased intensity. J Burn Care Res. 2013;34:94-101.

22. Yosipovitch G, Ansari N, Goon A, et al. Clinical characteristics of pruritus in chronic idiopathic urticaria. $\mathrm{Br} \mathrm{J}$ Dermatol. 2002;147:32-6.

23. Weisshaar E, Szepietowski JC, Darsow U, et al. European guideline on chronic pruritus. Acta Derm Venereol. 2012;92:563-81.

24. van der Zee HH, de Ruiter L, Boer J, et al. Alterations in leucocyte subsets and histomorphology in normal-appearing perilesional skin and early and chronic hidradenitis suppurativa lesions. Br J Dermatol. 2012;166:98-106.

25. Pascual JC, Garcia-Martinez FJ, Martorell A, et al. Increased total serum IgE levels in moderate-to-severe hidradenitis suppurativa. Br J Dermatol. 2016;175:1101-2.

26. Szepietowski JC, Reich A. Pruritus in psoriasis: an update. Eur J Pain. 2016;20:41-6.

27. Nattkemper LA, Martinez-Escala ME, Gelman AB, et al. Cutaneous T-cell lymphoma and pruritus: the expression of IL-31 and its receptors in the skin. Acta Derm Venereol. 2016;96(7):894-8.

28. Chuquilin M, Alghalith Y, Fernandez KH. Neurocutaneous disease: cutaneous neuroanatomy and mechanisms of itch and pain. J Am Acad Dermatol. 2016;74:197-212.

29. Halvorsen JA, Dalgard F, Thoresen M, et al. Itch and mental distress: a cross-sectional study among late adolescents. Acta Derm Venereol. 2009;89:39-44. 